\begin{tabular}{|c|l|}
\hline Title & Iterative angle and-time domain gating technique for time reversal MUSIC imaging \\
\hline Author(s) & Choi, Heedong; Ogawa, Y asutaka; Nishimura, Toshihiko; Ohgane, Takeo \\
\hline Citation & $\begin{array}{l}\text { Signal processing, 111, 39-49 } \\
\text { https://doi.org/10.1016/.sigpro.2014.12.007 }\end{array}$ \\
\hline Issue Date & 2015-06 \\
\hline Doc URL & http://hdl.handle.net/2115/58558 \\
\hline Type & article (author version) \\
\hline File Information & Ogawa_SP111_2015.pdf \\
\hline
\end{tabular}

Instructions for use 


\title{
Iterative Angle-and-Time-Domain Gating Technique for Time-Reversal MUSIC Imaging
}

\author{
Heedong Choi ${ }^{\mathrm{a}, 1}$, Yasutaka Ogawa ${ }^{\mathrm{a}, *}$, Toshihiko Nishimura ${ }^{\mathrm{a}}$, Takeo Ohgane ${ }^{\mathrm{a}}$ \\ ${ }^{a}$ Graduate School of Information Science and Technology, Hokkaido University, Sapporo 060-0814, Japan
}

\begin{abstract}
This study develops the preprocessing theory for time-reversal multiple signal classification (TR-MUSIC) imaging from the one-dimensional (time-domain) gating proposed in earlier papers to a two-dimensional one. Although the one-dimensional gating technique for TR-MUSIC mitigates the effect of noise as well as reduces the required number of antenna elements, the resulting image is subject to focusing errors when there are many targets in the gated region. In this paper, we propose a two-dimensional (angle-and-time-domain) gating technique based on the fast Fourier transform (FFT) and spatial FFT processing. By using these transforms and gating iteratively, we can reduce the number of targets to be detected at one time. The TR-MUSIC technique for the gated data provides more reliable images and precisely estimated coordinates compared with the previous work.

Keywords: Time reversal methodology, Multiple signal classification (MUSIC), Target detection, Radar imaging, Gating technique, Multistatic data matrix
\end{abstract}

\footnotetext{
*Corresponding author. Tel: +81 11706 7102; Fax: +81 117067102

Email address: ogawa@ist.hokudai .ac.jp (Yasutaka Ogawa)

${ }^{1}$ LG Electronics, Seoul, Korea (Present affiliation)
} 


\section{Introduction}

In recent years, numerous studies have attempted to devise remote sensing systems for detection and localization of obscured scatterers (targets) (e.g., [1-6]). In particular, the time reversal (TR) methodology [7, 8], which employs multipath components in the intervening media, provides better focusing resolution by increasing the effective aperture length virtually $[9,10]$. One of these methods, time-reversal multiple signal classification (TR-MUSIC), is a promising candidate for both single-frequency [11] and ultrawideband signals [12]. The TR-MUSIC algorithm was first proposed to overcome the resolution issue when there are more tranceivers $N$ than targets $M$ [11]. In the case of non-well-resolved (non-orthogonal) point-like targets, the conventional TR methodology does not work properly because of the mismatching of the eigenvalues with the scatterers [8]. By utilizing the noise subspace of the time reversal operator (TRO), which is orthogonal to the signal subspace, the TR-MUSIC algorithm has improved detection and imaging capabilities beyond those of the conventional method; i.e., it achieves super-resolution in the same sensing scenarios [11].

The TRO obtained from the multistatic data matrix (MDM) plays an essential role in TR-MUSIC imaging as well as in conventional TR techniques. For the most part, recent debate about TR has tended to center around the question of the organization and decomposition of the MDM, such as the matrices of the space-space, the space-frequency [13], the gated time-domain data [14], and the two-dimensional gated data [15]. The gating technique was originally proposed for electromagnetic wave measurements using a vector network analyzer [16-18]. A vector network analyzer measures reflection coefficients and/or transmission coefficients in the frequency domain. 
The effects of unwanted responses such as reflection from a connector of a device under test are almost always included in measured data. The frequency-domain measurement data are transformed into the time domain with an inverse fast Fourier transform (IFFT). If the frequency bandwidth is wide enough, the desired responses and unwanted responses are separated in the time domain. By using an adequate gate function that extracts only the desired responses, we can mathematically eliminate the unwanted responses in the time domain. After that, we apply a fast Fourier transform (FFT) to the gated time-domain data and obtain the frequency-domain data without the effects of the unwanted responses. This is the original gating technique. It has been reported that the gating technique is also useful for time-domain MUSIC because it can significantly reduce noise [19]. TR-MUSIC imaging with the time-domain gating that was proposed in earlier papers reduces noise by gating in the time domain and relaxes the antenna limitation by reducing the number of scatterers to be detected at one time [14]. It extracts the desired components (target responses) from the time-domain gated region; thus, the developed approach has a one-dimensional (time-domain) filtering effect in the scattering scenario. The "desired" components (responses) represent the signals in the gated region. Nevertheless, the image obtained from the one-dimensional gating is subject to focusing errors when there are many targets in the gated region. It should be noted that even though the number of the antennas is greater than that of the gated targets, TR-MUSIC cannot detect them properly in some cases. This is because the MDM obtained by finite snapshots is contaminated by noise. That is, TR-MUSIC only works well when there are a few scatterers. The gating scheme also requires a nontrivial condition that the target responses or the sidelobes (skirts) of the responses need 
to be separated from one another in order to extract the desired response(s) [14]. The above issues may be difficult to resolve because targets are irregularly and/or randomly distributed in the scattering area. These issues thus are the main motivation of this research.

To overcome the above obstacles, we have already proposed the basic concept of a joint angle-and-time-domain gating technique for TR-MUSIC imaging and have conducted a principal performance evaluation of two-dimensional (angle-and-timedomain) gating [15]. In this paper, we address the formulation of our approach in more detail, and we extend the two-dimensional processing for TR-MUSIC imaging based on iterative gating accompanied with an FFT and spatial FFT processing [20]. The ideas that form the spatial filtering concept are based on beamforming [21]. By adaptively weighting the received signals, the beamforming technique can estimate coarse directions of arrival. In addition, we can reduce the signals arriving outside the mainbeam. The developed TR-MUSIC algorithm based on one- or two-dimensional gating assumes the following conditions. First, the scatterers are located far apart from each other in the time domain because the time-domain gating technique must extract time-domain response(s) around a certain time without damaging the mainlobes and dominant sidelobes of the desired signal(s). Next, the scatterers are located far apart in the angle domain because of the limited number of array elements. Unfortunately, the scatterers are irregularly and/or randomly distributed over the scattering area and the number of antenna elements is limited. There is a significant disparity between the angle and time domains. That is, even when the signal responses are not resolved in the time domain, they sometimes can be classified in the angle domain. In this case, 
by utilizing the FFT and spatial FFT processing, we can easily identify each response and apply TR-MUSIC. Thus, if we eliminate unwanted responses in either the time or angle domain iteratively and complementarily, we can obtain a modified MDM that has contributions from fewer targets. The number of available dimensions of the noise subspace is an important consideration in TR-MUSIC imaging. Thus, it is preferable to decrease the number of targets to be detected at one time. Namely, if we remake a new MDM which includes fewer targets than those in the original data, we can suppress the above obstacles in the same scattering situation. The proposed approach is relatively simple, but it is quite effective at improving the performance of TR-MUSIC imaging by rebuilding modified MDMs. The remainder of this paper is divided into four main parts. The next section introduces the TR-based imaging technique using the MUSIC algorithm. Section 3 deals with the TR-MUSIC imaging with the gating technique and describes the proposed two-dimensional processing with iterative gating. Section 4 discusses the empirical evidence from computer simulations conducted to verify the effectiveness of this approach. Section 5 summarizes this study.

\section{Overview of TR based MUSIC Imaging}

As the name suggests, TR-MUSIC is an imaging technique combining the TR technique, which exploits the TR invariance of the wave equation, and MUSIC [11-13]. Suppose there are $N$ antenna elements of the time reversal array (TRA); TR-MUSIC imaging employs an $N \times N$ symmetric MDM, denoted as $\mathbf{K}(\omega)$, where $\omega$ is the angular frequency of the wave and bold letters denote vectors or matrices. Because the TR operation in the time domain is equivalent to phase conjugation in the fre- 
quency domain, $\mathbf{K}(\omega)$ can be expressed as $\mathbf{K}^{*}(\omega)=\mathbf{K}^{\dagger}(\omega)$ (due to the reciprocity principle), where the superscript asterisk ${ }^{*}$ denotes the complex conjugate and superscript ${ }^{\dagger}$ denotes the Hermitian conjugate. The TRO is defined as the self-adjoint matrix $\mathbf{T}(\omega)=\mathbf{K}^{\dagger}(\omega) \mathbf{K}(\omega)$. By applying singular value decomposition (SVD) to the MDM, we obtain $\mathbf{K}(\omega)=\mathbf{U}(\omega) \boldsymbol{\Lambda}(\omega) \mathbf{V}^{\dagger}(\omega)$, where $\mathbf{U}(\omega)$ and $\mathbf{V}(\omega)$ are unitary matrices, and $\boldsymbol{\Lambda}(\omega)$ is a diagonal and real-valued matrix of singular values $\lambda_{1}(\omega), \lambda_{2}(\omega), \ldots, \lambda_{N}(\omega)$.

Similarly, eigenvalue decomposition (EVD) of the TRO yields $\mathbf{T}(\omega)=\mathbf{V}(\omega) \mathbf{S}(\omega) \mathbf{V}^{\dagger}(\omega)$, where $\mathbf{S}(\omega)=\Lambda^{\dagger}(\omega) \Lambda(\omega)$ is a real-valued diagonal matrix with eigenvalues $\lambda_{1}^{2}(\omega), \lambda_{2}^{2}(\omega)$, $\ldots, \lambda_{N}^{2}(\omega)$. The eigenvectors of the TRO correspond to the columns of the unitary matrix $\mathbf{V}(\omega)$, which are composed of $\mathbf{v}_{i}(\omega)$ for $i=1,2, \ldots, N$. Thus, the signal subspace $\mathcal{S} S$ of the TRO is formed by eigenvectors $\mathbf{v}_{1}(\omega), \mathbf{v}_{2}(\omega), \ldots, \mathbf{v}_{M_{S}}(\omega)$ with significant eigenvalues $\lambda_{1}^{2}(\omega), \lambda_{2}^{2}(\omega), \ldots, \lambda_{M_{S}}^{2}(\omega)$, and the noise subspace $\mathcal{N S}$ is formed by $\mathbf{v}_{M_{S}+1}(\omega), \mathbf{v}_{M_{S}+2}(\omega), \ldots, \mathbf{v}_{N}(\omega)$, where $M_{S}$ is the number of significant eigenvalues. For point-like scatterers in homogeneous media, the number of targets $M$ is equal to the number of significant eigenvalues $M_{S}\left(M=M_{S}\right)$ [8]. When the signalto-noise ratio (SNR) is extremely high or there is almost no noise, the signal subspace $\mathcal{S S}$ is spanned by the eigenvectors having non-zero eigenvalues, whereas the noise subspace $\mathcal{N S}$ is spanned by the eigenvectors having almost zero eigenvalues, i.e., $\mathcal{S S}=\left\{\mathbf{v}_{1}(\omega), \mathbf{v}_{2}(\omega), \ldots, \mathbf{v}_{M}(\omega)\right\}$ with $\lambda_{1}^{2}(\omega) \geq \lambda_{2}^{2}(\omega) \geq \cdots \geq \lambda_{M}^{2}(\omega)>0$ and $\mathcal{N S}=\left\{\mathbf{v}_{M+1}(\omega), \mathbf{v}_{M+2}(\omega), \ldots, \mathbf{v}_{N}(\omega)\right\}$ with $\lambda_{M+1}^{2}(\omega) \approx \lambda_{M+2}^{2}(\omega) \approx \cdots \approx \lambda_{N}^{2}(\omega) \approx 0$.

Forming the image of the scatterers requires knowledge of the background Green's function vector, which is also called the background steering vector $\mathbf{g}\left(\mathbf{x}_{p}, \omega\right)=\left[G\left(\mathbf{x}_{p}, \boldsymbol{\alpha}_{1}, \omega\right)\right.$, $\left.G\left(\mathbf{x}_{p}, \boldsymbol{\alpha}_{2}, \omega\right), \ldots, G\left(\mathbf{x}_{p}, \boldsymbol{\alpha}_{N}, \omega\right)\right]^{T}$, where $\mathbf{x}_{p}$ and $\boldsymbol{\alpha}_{i}$ represent the search point vector and 
the position of the $i$ th antenna, respectively. Here, the superscript ${ }^{T}$ denotes the transpose. In homogeneous media, we have $G\left(\mathbf{r}, \mathbf{r}^{\prime}, \omega\right)=\left\{1 /\left(4 \pi\left\|\mathbf{r}-\mathbf{r}^{\prime}\right\|\right)\right\} \cdot \exp (-j \omega \| \mathbf{r}-$ $\mathbf{r}^{\prime} \| / c$ ), where $\mathbf{r}$ and $\mathbf{r}^{\prime}$ are the positions of the ends of a propagation path, $\|\cdot\|$ denotes the Euclidean norm, and $c$ denotes the propagation speed. Here, we assume that all antenna elements are isotropic and have the same and constant frequency characteristics of gain (or pattern) over the considered frequency band.

The Green's function vectors corresponding to the scatterers are orthogonal to the noise subspace $\mathcal{N S}$ because the noise subspace $\mathcal{N S}$ is always orthogonal to the signal subspace $\mathcal{S S}$. This condition is essentially the fundamental principle of the TR-MUSIC imaging algorithm and provides the following imaging function (imaging pseudo-spectra) expressed in the form $[11,12]$

$$
D\left(\mathbf{x}_{p}, \omega\right)=\left(\sum_{i=M+1}^{N} \mid<\mathbf{g}\left(\mathbf{x}_{p}, \omega\right), \mathbf{v}_{i}^{*}(\omega)>1\right)^{-1},
$$

where angular brackets $<\cdot>$ represent the inner product and $|\cdot|$ and the superscript ${ }^{-1}$ denote the absolute value and inverse, respectively.

\section{TR-MUSIC Imaging with Gating Technique}

In this section, we briefly review time-domain gating for TR-MUSIC imaging. After that, we elaborate on angle-domain gating and extend these schemes to the novel two-dimensional processing for TR-MUSIC imaging based on iterative gating accompanied with the FFT and spatial FFT processing. 


\subsection{Time-Domain Gating Technique}

TR-MUSIC imaging with time-domain gating was first proposed in [14]. As depicted in Fig. 1(a), a remote sensing system can be illustrated as a TRA consisting of $N$ antenna elements and $M$ scatterers. In this paper, we employ a single-frequency TR-MUSIC algorithm, and the imaging function is calculated with a single frequency as given by equation (1). We obtain, however, wide-band frequency-domain data in such a way that the scatterer responses are separable, and we can apply time-domain gating. In such an environment, the received signals in the time domain are represented as shown in Fig. 1(b). By applying the gating technique to the original data in the time domain, undesired factors (noise components and unwanted signals) can be mathematically eliminated. In the time-domain gating process, gate parameters such as the gate span (width), gate center, and gate type are determined depending on the scattering scenario. At this point, the gate must always include the desired target response(s) without damaging the mainlobe(s) and dominant sidelobes. The reason why each scatterer has sidelobes besides a mainlobe in the time domain is that the angular frequency bandwidth is finite, as stated in the following. Consider a case where sinusoidal waves are transmitted from antenna \#1 and the scattered waves from a single scatterer are received at the same antenna. We assume that the scatterer is located at the distance of $c t_{2}^{\prime} / 2$ from the antenna \#1 in homogeneous media, and that the measurement band is from $\omega_{c}-B / 2$ to $\omega_{c}+B / 2$. Here, $\omega_{c}$ and $B$ denote the central angular frequency and the angular frequency bandwidth, respectively. For simplicity, we also assume that the scattering coefficient is independent of angular frequency. Using the Green's function in homogeneous media given in Section 2, the frequency dependence of the 
phase of the received waves is $-\omega t_{2}^{\prime}$, and the IFFT of the waves at the antenna \#1 is $\left[\left\{\sin B\left(t-t_{2}^{\prime}\right) / 2\right\} /\left(t-t_{2}^{\prime}\right)\right] \cos \omega_{c}\left(t-t_{2}^{\prime}\right)$ to within a constant factor. Thus, the envelope of the time-domain response is given by $\left\{\sin B\left(t-t_{2}^{\prime}\right) / 2\right\} /\left(t-t_{2}^{\prime}\right)$. The peak around $t=t_{2}^{\prime}$ is the mainlobe, and the peaks around $t=t_{2}^{\prime} \pm(2 n+1) \pi / B(n=1,2,3, \ldots)$ are sidelobes. Since the angular frequency bandwidth is finite, we have sidelobes in addition to the mainlobe.

Let us assume that $M \geq N$ holds. In this case, TR-MUSIC does not work since it employs the noise subspace, as stated in the previous section. This is the limitation of the antenna structure. If the time-domain gate range is from $t_{0}^{\prime}$ to $t_{0}^{\prime}+\tau$, we only have scatterers located from $c t_{0}^{\prime} / 2$ to $c\left(t_{0}^{\prime}+\tau\right) / 2$, as shown in Fig. 1(a). If the number of scatterers in this region is less than $N$, the limitation can be relaxed by gating. Also, if we use a rectangular gate function with span $T_{g}$, noise outside the gate is eliminated. Because the distribution of noise is statistically uniform in the time domain, the noise power is reduced by $T_{g} /(1 / \delta f)=T_{g} \delta f$, where $\delta f$ is the sampling frequency separation. The noise reduction is also possible with other gating functions. Therefore, TR-MUSIC imaging with time-domain gating not only relaxes the limitation on the antenna structure but also mitigates the effect of noise. The gate span $T_{g}$ is determined in such a way that the gate includes the mainlobes and dominant sidelobes of the responses in this region. This results in an enhancement in the SNR. In general, the higher SNR is, the more precisely all the MUSIC algorithms detect signals since we have a more precise noise subspace. This is also the case for TR-MUSIC. With the higher SNR, TR-MUSIC can precisely detect point-like scatterers, and this improves the imaging quality. 


\subsection{Angle-Domain Gating Technique}

Here, we describe angle-domain gating for TR-MUSIC imaging to extract scatterer(s) in the angle domain. The technique is based on the spatial FFT.

The concept of the spatial FFT is described in relation to the beamforming technique in [21]. By using it, we can obtain the angle-domain data for received signals. The conventional beamforming matrix (or weight matrix) $\mathbf{W}$ is essentially based on the Fourier matrix. Because the number of transceivers is $N$, we have an $N \times N$ beamforming matrix whose $m$ th row vector $\mathbf{w}_{m}(\omega)$ for the angular frequency $\omega$ is given by

$$
\mathbf{w}_{m}(\omega)=\left[w^{0}(\omega), w^{m}(\omega), w^{2 m}(\omega), \ldots, w^{(N-1) m}(\omega)\right],
$$

where $w(\omega)=\exp \left[(-j 2 \pi / N)\left(\omega / \omega_{c}\right)\right]$ and $m=0,1, \ldots, N-1$.

However, if we let the angle of the array normal be 0 degrees, the angle-domain data obtained from the above conventional beamforming equation has a phenomenon wherein the mainbeam direction suddenly changes from $90^{\circ}$ to $-90^{\circ}$ : i.e., the mainbeam direction is a sequence of $\left(0^{\circ}, 1^{\circ}, 2^{\circ}, \ldots, 90^{\circ},-90^{\circ},-89^{\circ}, \ldots,-2^{\circ},-1^{\circ}\right)$. To avoid this problem, we can modify the $N \times N$ beamforming matrix as follows: 


$$
\begin{aligned}
& \mathbf{W}^{\prime}(\omega)= \\
& \left(\begin{array}{ccccc}
w^{0}(\omega) & w^{\left(-\frac{N}{2}+1\right)}(\omega) & w^{2\left(-\frac{N}{2}+1\right)}(\omega) & \cdots & w^{(N-1)\left(-\frac{N}{2}+1\right)}(\omega) \\
w^{0}(\omega) & w^{\left(-\frac{N}{2}+2\right)}(\omega) & w^{2\left(-\frac{N}{2}+2\right)}(\omega) & \cdots & w^{(N-1)\left(-\frac{N}{2}+2\right)}(\omega) \\
\vdots & \vdots & \vdots & \ddots & \vdots \\
w^{0}(\omega) & w^{-1}(\omega) & w^{-2}(\omega) & \cdots & w^{-(N-1)}(\omega) \\
w^{0}(\omega) & w^{0}(\omega) & w^{0}(\omega) & \cdots & w^{0}(\omega) \\
w^{0}(\omega) & w^{1}(\omega) & w^{2}(\omega) & \cdots & w^{(N-1)}(\omega) \\
\vdots & \vdots & \vdots & \ddots & \vdots \\
w^{0}(\omega) & w^{\frac{N}{2}}(\omega) & w^{2\left(\frac{N}{2}\right)}(\omega) & \cdots & w^{(N-1)\left(\frac{N}{2}\right)}(\omega)
\end{array}\right)
\end{aligned}
$$

Here, we have assumed that $N$ is even. We express the $m$ th row vector of $\mathbf{W}^{\prime}(\omega)$ as $\mathbf{w}_{m}^{\prime}(\omega)$ where the index $m$ has values $-N / 2+1,-N / 2+2, \ldots, N / 2$. In the remainder of this paper, we will assume that the antenna spacing is a half wavelength $\lambda_{c} / 2$ corresponding to the central frequency.

Figure 2 shows the first and the second antenna elements for the ULA, and their complex weights $w^{0}(\omega)=1$ and $w^{m}(\omega)=\exp \left[(-j 2 \pi m / N)\left(\omega / \omega_{c}\right)\right]$. The angle $\theta$ is measured clockwise from the array normal. In this case, the path difference $d$ and the delay difference $\tau$ between adjacent antenna elements are given by

$$
\begin{gathered}
d=\lambda_{c} \sin \theta / 2 \\
\tau=\frac{d}{c}=\frac{\pi \sin \theta}{\omega_{c}} .
\end{gathered}
$$


As a result, the phase difference $\phi(\omega)$ at angular frequency $\omega$ is given by

$$
\phi(\omega)=\omega \tau=\frac{\pi \omega}{\omega_{c}} \sin \theta
$$

As seen from Fig. 2, we have the mainbeam for the direction in which $\phi(\omega)=$ $(2 \pi m / N)\left(\omega / \omega_{c}\right)$ holds. Thus, the mainbeam direction is given by

$$
\begin{gathered}
\sin \theta_{0}=\frac{2 m}{N} \\
\theta_{0}=\sin ^{-1}\left(\frac{2 m}{N}\right) .
\end{gathered}
$$

However, there is still an impact from high sidelobes that result from the uniform amplitude. The amplitude of all the weights in equations (2) and (3) is 1, namely, uniform. This corresponds to that we employ a rectangular widow function before the spatial FFT, and the rectangular window function generates high sidelobes. To reduce the sidelobe levels, we can instead utilize a Gaussian window function, which has the least effect on the sidelobes among typical window functions having a non-zero value at both ends of the region. The effect can be seen in Fig. 3, which shows the amplitudes of the weights for $N=64$ and the corresponding patterns $(m=0)$ for different window functions. Here, we only considered the window functions having non-zero values such as the rectangular, Hamming, and Gaussian window functions because we need an inverse matrix calculation after gating in the angle domain. The rectangular window function causes relatively higher sidelobes compared with the other window functions. The modified beamforming matrix whose $m$ th row vector $\mathbf{w}_{m}^{\prime \prime}$ is given by (After this point, we will omit the angular frequency variable $\omega$ for simplicity.) 


$$
\mathbf{w}_{m}^{\prime \prime}=\mathbf{w}_{m}^{\prime} \mathbf{Z}
$$

where $\mathbf{Z}$ is an $N \times N$ diagonal matrix whose diagonal elements are given by a Gaussian function. Although the modified beamforming matrix $\mathbf{W}^{\prime \prime}$ with row vectors $\mathbf{w}_{m}^{\prime \prime}$ given by (9) is different from the FFT matrix $\mathbf{W}^{\prime}$, we consider the transform using $\mathbf{W}^{\prime \prime}$ to be the spatial FFT.

Regarding angle-domain gating, we apply the spatial FFT to the original MDM $\mathbf{K}(\omega)$ and obtain the angle-domain data $\mathbf{K}(\theta)=\mathbf{W}^{\prime \prime} \mathbf{K}(\omega)$. Although $\mathbf{K}(\theta)$ is also a function of $\omega$, we will omit it in the equations for simplicity. Next, we apply the gating to each column of $\mathbf{K}(\theta)$ to extract the response(s) from a certain angle region. This process is basically the same as time-domain gating. Time-domain gating can be easily obtained through a large number of sampling points by utilizing wideband data. On the other hand, the number of antennas is limited. Thus, angle-domain gating must deal with much fewer sampling points than in the time-domain case. Consequently, we pad elements other than those of the directions of interest with zeros. After the angle-domain gating, we obtain the angle-domain gated $\operatorname{MDM} \mathbf{K}^{\prime}(\theta)$. Because we use a Gaussian window function for the modified beamforming matrix $\mathbf{W}^{\prime \prime}$, the matrix $\mathbf{W}^{\prime \prime}$ is not unitary any more, i.e., $\mathbf{W}^{\prime \prime-1} \neq \mathbf{W}^{\prime \prime \dagger}$. Thus, we return to the MDM as follows: $\mathbf{K}^{\prime}(\omega)=\mathbf{W}^{\prime \prime-1} \mathbf{K}^{\prime}(\theta)$. As shown in Figs. 1(d) and (e), if the angle-domain gate range is from $\theta_{0}^{\prime}$ to $\theta_{0}^{\prime}+\xi$, we only have scatterers located in the corresponding directions. Thus, by using this technique, we can extract the desired response(s) in the angle directions and relax the antenna limitation. The angle-domain gating, namely, zero padding, suppresses not only scatterer responses but also noise components outside the range, and 
the SNR improves as a result. However, the noise reduction effect is smaller than in the time-domain case because of the limited number of sampling points.

\subsection{Two-Dimensional Processing based on Iterative Gating}

Consider the remote sensing system example illustrated in Fig. 4. Let us assume that there are $M$ point-like scatterers and $N$ antenna elements and that multiple scattering is negligibly small. In such an environment, each TRA element receives $M$ signal responses which correspond to the reflected signals from all the scatterers. In case multiple scattering signals are not negligible, TR-MUSIC will detect false scatterers located farther than true ones because multiple scattering signals have longer roundtrip time. Although we do not treat multiple scattering in this paper, it is an important issue to be solved in future.

Although the gating techniques can potentially improve performance, each technique has a limit. As stated previously, if the snapshots are limited, TR-MUSIC works well when there are only a few scatterers. If we have many scatterers in either the time-domain gating region or in the angle-domain one, TR-MUSIC cannot find their locations. This leads us to the iterative gating scheme described below.

Since there are many more sampling points in the time domain than in the angle domain, we first obtain the time-domain response. If the gate region for the timedomain gating is from $t_{0}^{\prime}$ to $t_{0}^{\prime}+\tau_{1}$, we obtain scatterers located in the range from $c t_{0}^{\prime} / 2$ to $c\left(t_{0}^{\prime}+\tau_{1}\right) / 2$, as shown in Fig. 4 (Step 1). As mentioned in the previous subsection, the noise components outside the gate are suppressed in this step. Next, we apply the FFT and spatial FFT to obtain the angle-domain data and observe the arrival angles in the angle domain. The undesired signals outside the gate are suppressed by using 
angle-domain gating from $\theta_{0}^{\prime}$ to $\theta_{0}^{\prime}+\xi_{1}$ (Step 2). Note that the angle-domain gating process is fundamentally equivalent to the time-domain one. After the angle-domain gating, the time-domain response has changed. Thus, we apply the spatial IFFT and IFFT to the angle-and-time-domain gated data. We then extract the time-domain signal response(s) by using time-domain gating from $t_{0}^{\prime}+\tau_{2}$ to $t_{0}^{\prime}+\tau_{3}$ (Step 3). Similarly, we transform the gated data to angle-domain data and extract the desired scatterer(s) from $\theta_{0}^{\prime}+\xi_{2}$ to $\theta_{0}^{\prime}+\xi_{3}$ by utilizing angle-domain gating (Step 4). We repeat this process until there are only a few signals in one of the domains. The gate region is important both in the time domain and in the angle domain. We determine the region in such a way that the gate function includes the desired mainlobe(s) and dominant sidelobes. At present, we manually determine the region. After that, we apply TR-MUSIC imaging. By changing the gating region, we can detect targets located around the array normal. If we move the TRA mechanically and apply the process, we can find all the targets. Since the proposed approach reduces noise and increases the number of noise subspaces, it improves the performance of TR-MUSIC imaging.

\section{Simulation Results and Discussions}

We conducted computer simulations of our scheme. The following scenario was used to evaluate the imaging performance. The frequency band was assumed to be from $1.5 \mathrm{GHz}$ to $3.5 \mathrm{GHz}$ (central frequency $f_{c}=2.5 \mathrm{GHz}$, and the sampling frequency separation was $\delta f=10 \mathrm{MHz}$ ). The linear TR array consisted of $N=32$ isotropic antennas with the gain of $0 \mathrm{dBi}$ and was placed on the $\mathrm{x}$-axis. The 16 th antenna element was located at the origin of the coordinates. Twenty-five point-like scatterers $(M=25)$ 
with identical scattering coefficients $\rho_{m}=1$ in the measurement frequency band ( $m$ $=1,2, \ldots, 25)$ were irregularly distributed on the Cartesian coordinate system, as shown in Fig. 5. In a case where scattering coefficients are frequency-dependent, we may encounter a problem. This issue needs to be considered in future. The simulations were conducted in additive zero mean Gaussian noise so as to have a SNR of $30 \mathrm{~dB}$. Here, the signal power was defined as that of reflection from the target at $(-3.5 \mathrm{~m}$, $2.5 \mathrm{~m})$. The imaging function for the TR-MUSIC scheme was obtained for the center frequency with a single snapshot. Note that we have different results depending on the environment, i. e., depending on the SNR value and/or scatterer locations. If the SNR is lower, we will have poorer performance than that stated below.

Figure 6 shows the time-domain responses of $k_{16,16}(t)$ and the angle-domain responses of the first column vector of MDM for the raw and gated data. Here, the MDM element $k_{i, j}$ corresponds to the signal received at the $i$ th antenna when the $j$ th antenna transmits the signal. As can be seen from Fig. 6(a), there are many peaks corresponding to the scatterers, complicatedly ranging from around 20 to $70 \mathrm{~ns}$. In addition, there is propagation attenuation. By applying the spatial FFT to the raw frequencydomain data, we obtained the raw angle-domain data shown in Fig. 6(b). Since we used a ULA with 32 antenna elements, the mainbeam directions for the $m$ th row of the matrix $\mathbf{W}^{\prime \prime}$ are $\sin ^{-1}(2 m / 32)$ for $m=-15,-14, \ldots,-1,0,1, \ldots, 15,16$. The indexes $(m=-15,-14, \ldots,-1,0,1, \ldots, 15,16)$ correspond to the angles. As depicted in Fig. 6(b), the scatterers are widely distributed in all directions, and the normalized values in the angle domain range from 0.11 to 1 . This shows that the angle-domain gating technique cannot be directly applied to this data. 
We focused on the scatterer with the delay time of $40 \mathrm{~ns}$ and located around the array normal. As seen from Fig 6 (a), several mainlobes are separated at short time intervals from about $40 \mathrm{~ns}$ to $60 \mathrm{~ns}$, and their sidelobes overlap. As mentioned in Section 3, the gate function must include both the desired scatterers' mainlobes and the dominant sidelobes. We need to extract all the above responses by using the time-domain gating. We applied the time-domain gating to the original raw data and obtained the first gated data in the time domain, as shown in Fig. 6(c). The gate parameters were as follows: gate center $T_{0}=49 \mathrm{~ns}$, gate span $T_{g}=32 \mathrm{~ns}$, and raised cosine gate function with a roll-off $=0.2$. We examined several gating functions. Among them, the raised cosine gate function gave the best result. This is why we employed the above parameters. After the time-domain gating, the angle-domain response was obtained by applying the FFT and spatial FFT to the gated data, as depicted in Fig. 6(d). The first gated data in the angle domain takes on a different aspect compared with the raw data shown in Fig. 6(b). One can see that there are three groups of targets. One is around the array normal region from about $-30^{\circ}$ to $30^{\circ}$. The others are around the array axis regions from about $30^{\circ}$ to $90^{\circ}$, and from about $-30^{\circ}$ to $-90^{\circ}$. This situation enables us to perform angle-domain gating. We applied the angle-domain gating to eliminate the target responses around the directions in the array axis. We can see that the responses at the indexes -8 and 8 have a very small value, and the above three groups are separated at -8 and 8 . As mentioned in Section 3, we padded zeros from the index -15 to -9 and from 9 to 16 (Fig. 6(e)). The regions correspond to angles from $-70^{\circ}$ to $-34^{\circ}$ and from $34^{\circ}$ to $90^{\circ}$, respectively. After performing the spatial IFFT and IFFT, we obtained the second gated data in the time domain (Fig. 6(f)). As can be seen in this figure, the 
time-domain response has fewer signals than in Fig. 6(c) because only the scatterers located around the array normal were extracted by the angle-domain gating. We again applied time-domain gating to the second gated data and extracted the signal located at $40 \mathrm{~ns}$ (Fig. 6(g)). The gate parameters for extracting the scatterer are a gate center $T_{0}=40 \mathrm{~ns}$, gate span $T_{g}=12 \mathrm{~ns}$, and raised cosine gate function with a roll-off $=$ 1. The best way to gate the signal response is to extract the mainlobe and dominant sidelobes without damaging them. Therefore, a gate function with a gentle slope is preferred. This is why we employed a different roll-off factor from the previous one. Figure 6(h) shows the angle-domain response, which concentrates in the direction of the array normal. The time- and angle-domain responses for the third gated data reveal that we cannot apply the gating technique anymore.

Next, let us consider the effect of the iterative angle-and-time-domain gating technique using the singular value distributions shown in Fig. 7. The presented values were obtained from the MDM at a central frequency $f_{c}=2.5 \mathrm{GHz}$ and are normalized in such a way that the maximum value in each case is 1 . When we utilize a subspace-based algorithm such as MUSIC, we need to know in advance the number of dimensions of the noise subspace $D_{n}$. Although the theoretical number of dimensions of the noise subspace is $7(N=32, M=25)$ for the raw data, there are 12 values that are almost zero (Fig. 7(a)). This means that the singular value distribution is not well-suited for detecting all the targets using the original raw data. Although the number of responses can not be clearly seen in Fig. 6(c), the time-domain gated region for the first process includes 14 scatterers. Thus, we can eliminate 11 scatterers, and the theoretical number of dimensions of the noise subspace is $18(N=32, M=14)$. However, there are 20 
singular values having very small values (Fig. 7(b)). After the second gating process, the theoretical number of dimensions of the noise subspace is $28(N=32, M=4)$ because the gated region from the sequential process has only four scatterers. As can be seen from Fig. 7(c), 28 of the singular values are very small. Similarly, after the third gating process, we can obviously identify one singular value having a much larger value than the others (Fig. 7(d) $(N=32, M=1))$. In the third gated region, we have only one scatterer. The number of non-zero singular values is consistent with the scattering situation. We can therefore expect that the iterative gating technique improves TR-MUSIC's imaging performance.

Figure 8 shows the pseudo-spectra of TR-MUSIC imaging with raw and gated data. Here, the white circles represent the true coordinates of the scatterers. The dimensions of the noise subspace are 12, 20, 28 and 31 for Figs. 8(a), (b), (c) and (d), respectively. As can be seen from Fig. 8(a), the estimated coordinates, as well as the resulting images, have been damaged and twisted by the noise components and many scatterers. As mentioned above, the number of dimensions of the noise subspace is not valid. The noise conditions, as well as the dimension of the noise subspace, affect TR-MUSIC imaging. Therefore, performance degradation is inevitable if we use the raw data. The TR-MUSIC pseudo-spectra image shown in Fig. 8(b) was obtained from the first gated data. Compared with the raw data case, the first gated data is different. However, it is still difficult to identify scatterers in spite of the time-domain gating technique. As stated previously, we used the raised cosine gate function with the gate center of $49 \mathrm{~ns}$ and the gate span of $32 \mathrm{~ns}$, and the roll-off parameter was 0.2. The gated range was almost from $33 \mathrm{~ns}$ to $65 \mathrm{~ns}$. The targets outside the range from $4.95 \mathrm{~m}$ to $9.75 \mathrm{~m}$ were 
almost suppressed with the time-domain gating. We applied, however, TR-MUSIC to the whole region to examine the imaging scheme. We see that there are some target responses outside the gated range, and that TR-MUSIC does not work properly at this stage. This is important information when we apply TR-MUSIC. The reason why some target responses appeared outside the gated range can be explained as follows. Even after the gating process, there were as many as 14 targets in the range, and these many targets and noise invited errors. Moreover, the TR-MUSIC imaging function Eq. (1) has values over a certain range. Thus, some responses of TR-MUSIC appeared also outside the gated range. Figure 8(c) shows the pseudo-spectra of TR-MUSIC imaging with the second gated data. We can see the effect of the gating process. Although the two gating steps reduce the noise and number of scatterers to be detected, the TR-MUSIC pseudo-spectra show deformed images with erroneous coordinates. TRMUSIC should work properly in this case because we have much fewer targets than the number of antenna elements and the dimension of the noise subspace is correct. We have, however, residual noise, and the noise degrades the performance of TR-MUSIC when targets are closely located as in this case.

Finally, from Fig. 8(d), we can see that our approach can precisely estimate the location of the desired scatterer and provide a clearer image function in the same SNR environment. Thanks to the noise reduction and relaxation of the transceiver limitation, our approach increases the SNR and leads to better imaging performance. In addition, by applying different time-domain gates to the data shown in Fig. 8(c), we can detect other targets. As for the targets located around the array axis directions, we need to move the TRA along the $\mathrm{x}$-axis in such as way that they are located around the array 
normal directions. As stated previously, if the SNR is higher, we can detect and resolve more closely located scatterers since we have a more precise noise subspace.

\section{Conclusions}

This paper proposed a new two-dimensional (angle-and-time-domain) iterative gating technique for use in conjunction with the TR-based imaging/detection algorithm. Although the original TR-MUSIC algorithm provides superior imaging and detection capabilities, it has the prerequisite as to the antenna structure and necessitates many sets of frequency-domain data in noisy environments. To overcome the above obstacles, the one-dimensional (time-domain) approach had been proposed and developed by the authors. The one-dimensional approach suppresses the effect of noise as well as reduces the required number of antenna elements in limited scattering situations by eliminating undesired components in the time domain. Unfortunately, the technique is subject to focusing errors when there are many targets that are irregularly and/or randomly distributed in the gated region. The approach presented in this paper, on the other hand, extends the scheme for TR-MUSIC imaging from one-dimensional gating to two-dimensional one. By iteratively and complementarily utilizing time- and angledomain gating, we can obtain a modified MDM that has contributions from fewer targets and lower noise in the same scattering situation. This leads to an increase in SNR and improves imaging performance by exploiting each domain's strengths. In this paper, the gate parameters were determined depending on situation. Their optimization should be studied in future. In this paper, we assumed point-like targets. As for extended targets, it is considered that TR-MUSIC detects specular reflection points on 
them. This issue will also be a subject of future work.

\section{References}

[1] T. G. Savelyev, L. van Kempen, H. Sahli, J. Sachs, M. Sato, Investigation of time-frequency features for GPR landmine discrimination, IEEE Trans. Geosci. Remote Sens. 45 (2007) 118-129. doi:10.1109/TGRS.2006.885077.

[2] A. C. Gurbuz, J. H. McClellan, W. R. Scott, A compressive sensing data acquisition and imaging method for stepped frequency GPRs, IEEE Trans. Signal Process. 57 (2009) 2640-2650. doi:10.1109/TSP.2009.2016270.

[3] F. Foroozan, A. Asif, Time-reversal ground-penetrating radar: Range estimation with Cramér-Rao lower bounds, IEEE Trans. Geosci. Remote Sens. 48 (2010) 3698-3708. doi:10.1109/TGRS.2010.2047726.

[4] Y. S. Yoon, M. G. Amin, Spatial filtering for wall-clutter mitigation in throughthe-wall radar imaging, IEEE Trans. Geosci. Remote Sens. 47 (2009) 3192-3208. doi:10.1109/TGRS.2009.2019728.

[5] W. Zhang, A. Hoorfar, L. Li, Through-the-wall target localization with time reversal MUSIC method, Progress In Electromagnetics Research 106 (2010) 75-89. doi:10.2528/PIER10052408.

[6] S. Xin, L. Biying, Z. Yang, Z. Lanzi, Z. Zhimin, Wall artifacts removal for target imaging enhancement in UWB through-the-wall radar application, Signal Processing 104 (2014) 325-338. doi:10.1016/j.sigpro.2014.04.021. 
[7] C. Prada, J. L. Thomas, M. Fink, The iterative time reversal process: Analysis of the convergence, J. Acoust. Soc. Amer. 97 (1995) 62-71. doi:10.1121/1.412285.

[8] C. Prada, S. Manneville, D. Spoliansky, M. Fink, Decomposition of the time reversal operator: Detection and selective focusing on two scatterers, J. Acoust. Soc. Amer. 99 (1996) 2067-2076. doi:10.1121/1.415393.

[9] P. Blomgren, G. Papanicolaou, H. Zhao, Super-resolution in time-reversal acoustics, J. Acoust. Soc. Amer. 111 (2002) 230-248. doi:10.1121/1.1421342.

[10] M. E. Yavuz, F. L. Teixeira, Ultrawideband microwave sensing and imaging using time-reversal techniques: A review, Remote Sens. 9 (2009) 466-495. doi: $10.3390 /$ rs 1030466 .

[11] A. J. Devaney, Time reversal imaging of obscured targets from multistatic data, IEEE Trans. Antennas Propag. 53 (2005) 1600-1610. doi:10.1109/TAP.2005.846723.

[12] M. E. Yavuz, F. L. Teixeira, On the sensitivity of time-reversal imaging techniques to model perturbations, IEEE Trans. Antennas Propag. 56 (2008) 834-843. doi:10.1109/TAP.2008.916933.

[13] M. E. Yavuz, F. L. Teixeira, Space-frequency ultrawideband time-reversal imaging, IEEE Trans. Geosci. Remote Sens. 46 (2008) 1115-1124. doi:10.1109/TGRS.2008.915755.

[14] H. Choi, Y. Ogawa, T. Nishimura, T. Ohgane, Time-reversal MUSIC imaging 
with time-domain gating technique, IEICE Trans. Commun. E95-B (2012) $2377-$ 2385. doi:10.1587/transcom.E95.B.2377.

[15] H. Choi, Y. Ogawa, T. Nishimura, T. Ohgane, Angle-and-time-domain gating technique for time-reversal MUSIC imaging, Proc. ISAP (International Symposium on Antennas and Propagation), Nagoya, Japan, 2012, pp. 86-89.

[16] W. Briggs, Analyzer improvements make real-time antenna tests practical, MSN \& CT, (1987) 74-86.

[17] J. W. Boyles, Long range antenna measurements with the HP8510 using harmonic mixers, Proc. AMTA (Antenna Measurement Technique Association) Eighth Annual Meeting and Symposium, Ottawa, Canada, 1986, pp. 19-24.

[18] H. Yamada, M. Ohmiya, Y. Ogawa, K. Itoh, Superresolution techniques for timedomain measurements with a network analyzer, IEEE Trans. Antennas Propag. 39 (1991) 177-183. doi:10.1109/8.68179.

[19] Y. Ogawa, T. Nakajima, H. Yamada, K. Itoh, A superresolution technique for antenna pattern measurements, IEICE Trans. Commun. E76-B (1993) 1532-1537.

[20] H. Choi, Y. Ogawa, T. Nishimura, T. Ohgane, Sequential gating processing for time-reversal MUSIC, IEICE Tech. Rep. A.P 2012-33 112 (2012) 17-22, (without peer review).

[21] J. Litva, T. K. Y. Lo, Digital beamforming in wireless communications, Artech House, Norwood, MA, 1996, pp. 13-56. 


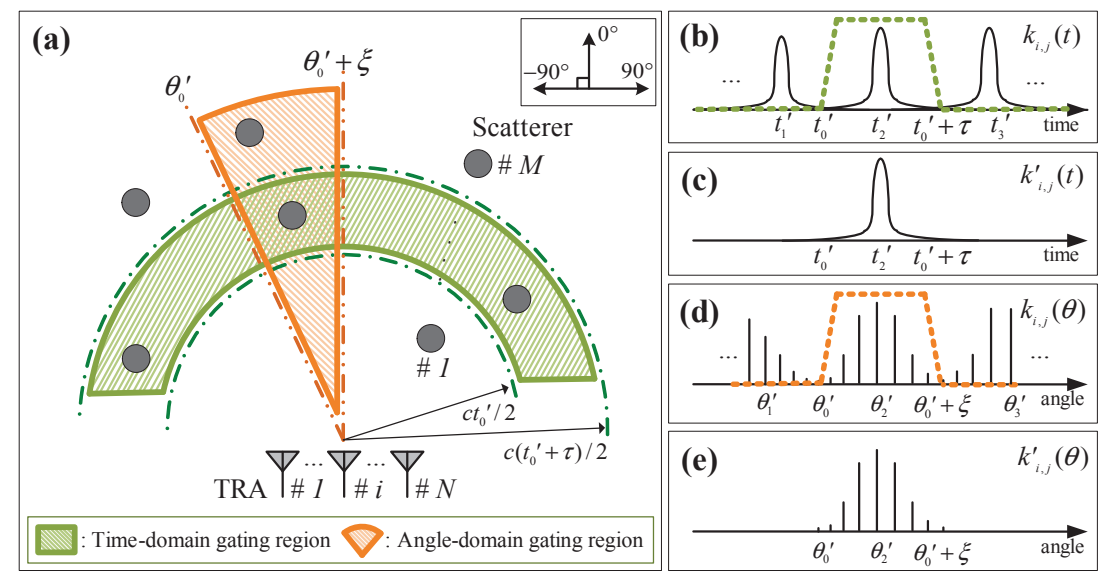

Figure 1: Concept of the time- and angle-domain gating technique. (a) schematic illustration of the remote sensing system (b) raw time-domain data (c) gated time-domain data (d) raw angle-domain data (e) gated angle-domain data. 


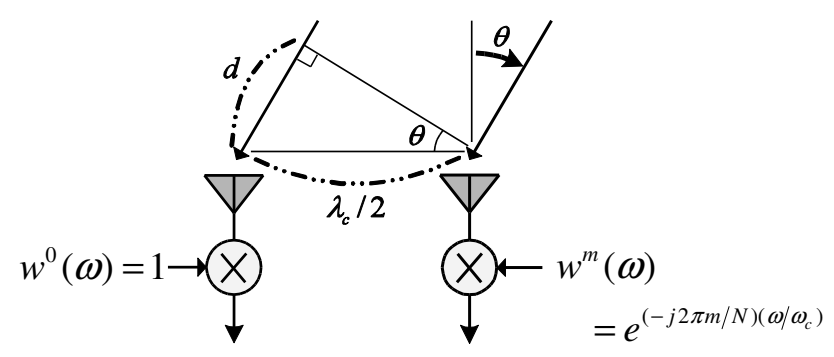

Figure 2: Weights for adjacent antenna elements. The angle $\theta$ is measured clockwise from the array normal. 


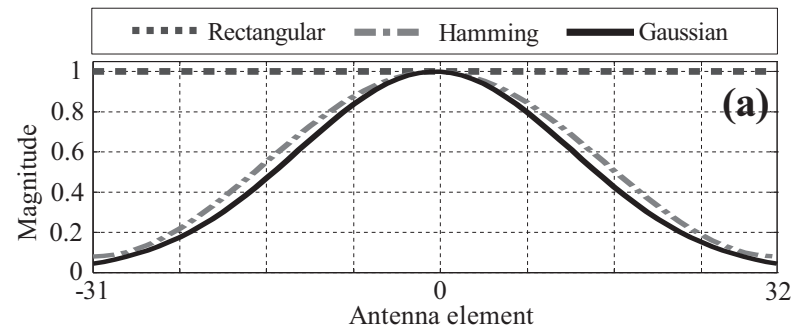

(a) amplitudes of weights

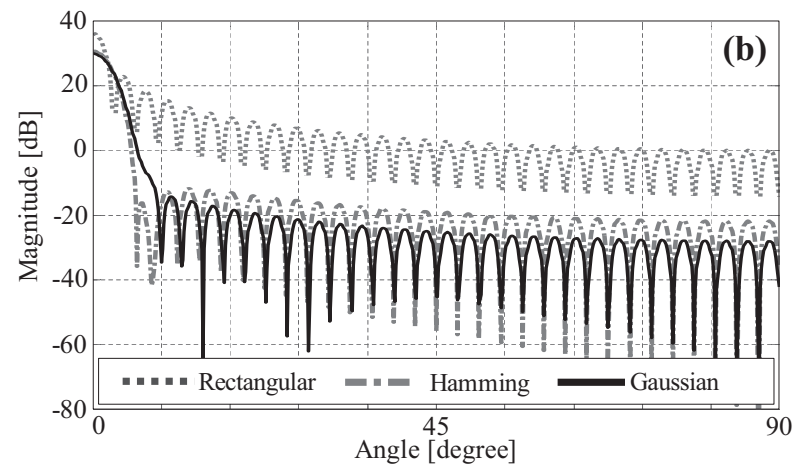

(b) patterns for $m=0$.

Figure 3: Amplitudes of weights and patterns for different window functions. 


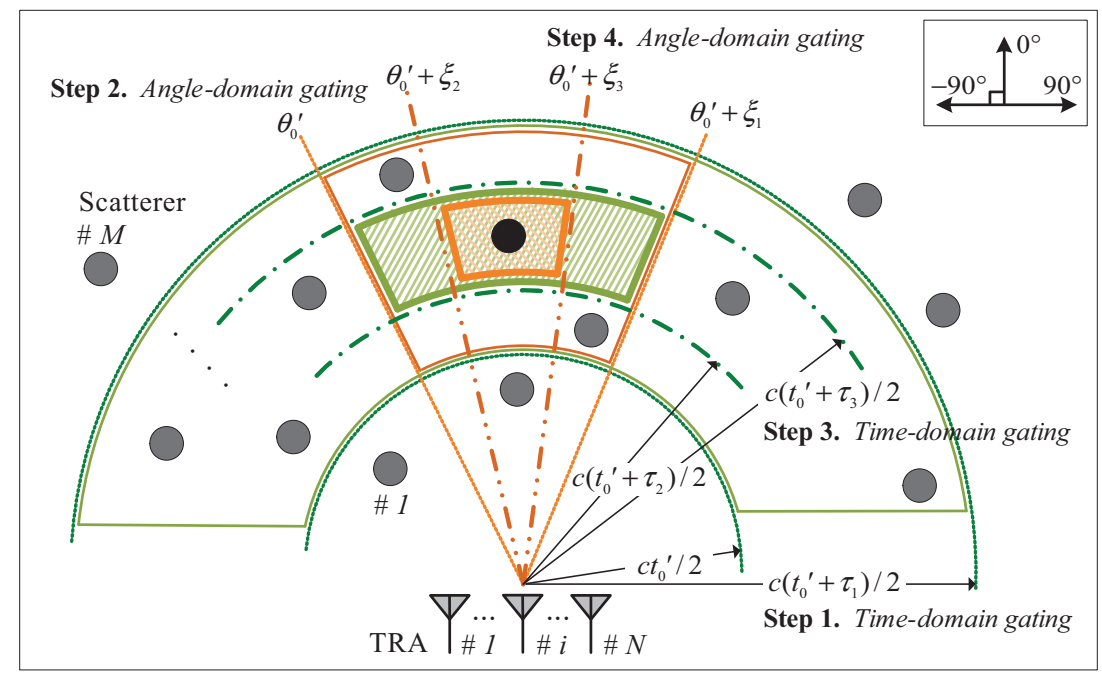

Figure 4: Concept of two dimensional processing based on iterative gating. Time-domain gating is applied first because there are many samples in the time domain. After that, angle-domain gating is applied to the gated data. This process continues until we only have a few signals in one of the domains. 


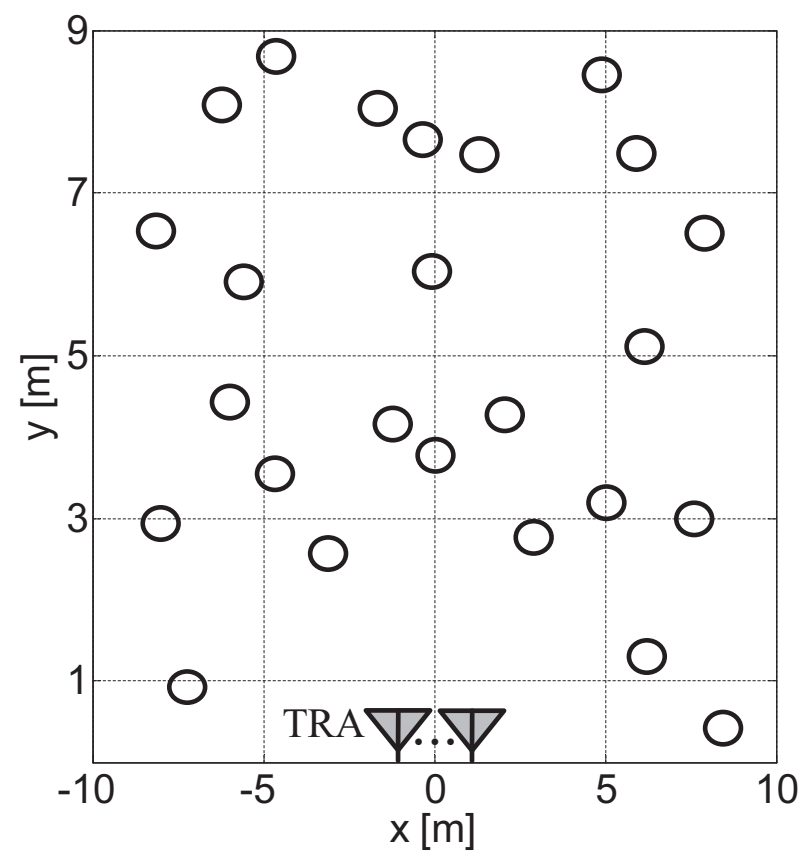

Figure 5: TRA and scatterer locations. The circles indicate the scatterers. 

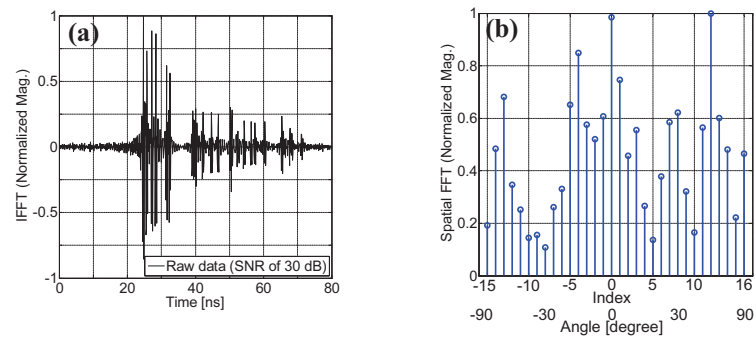

$(\mathrm{a}, \mathrm{b})$ original raw data
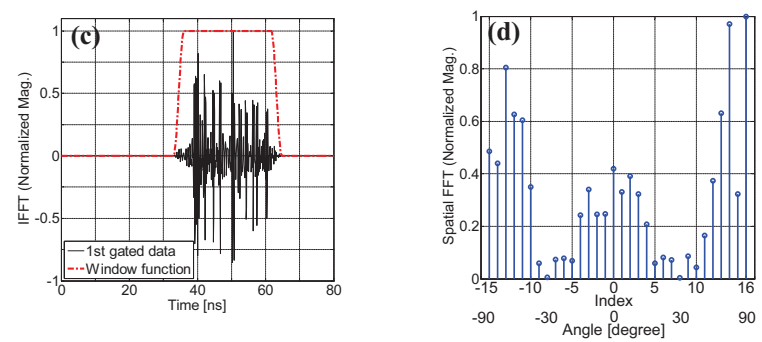

(c, d) first gated data $\left(T_{0}=49 \mathrm{~ns}, T_{g}=32 \mathrm{~ns}\right)$
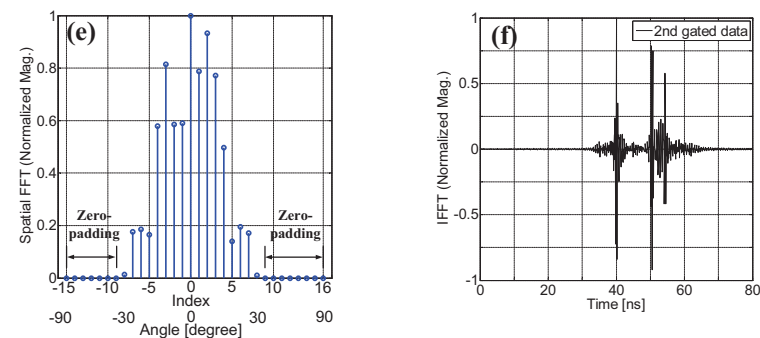

$(e, f)$ second gated data (indexes of zero-padding angle-domain region: -15- $-9,9-16$ )
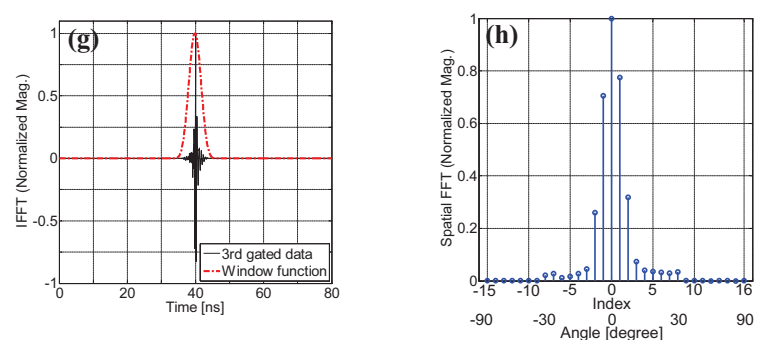

$(\mathrm{g}, \mathrm{h})$ third gated data $\left(T_{0}=40 \mathrm{~ns}, T_{g}=12 \mathrm{~ns}\right)$

Figure 6: Signal responses in the time and angle domains. 


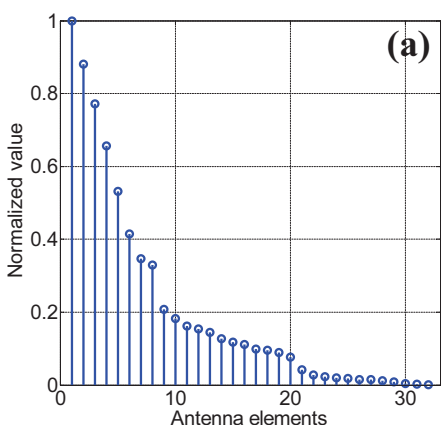

(a) raw data $\left(D_{n}=12\right)$

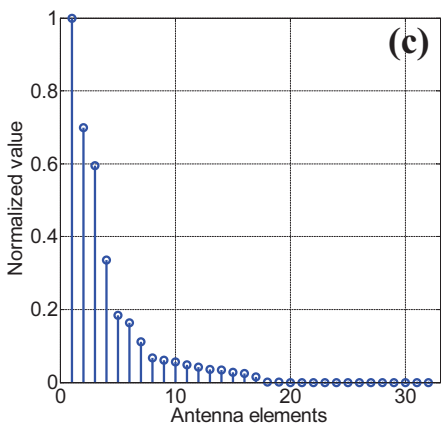

(c) second gated data $\left(D_{n}=28\right)$

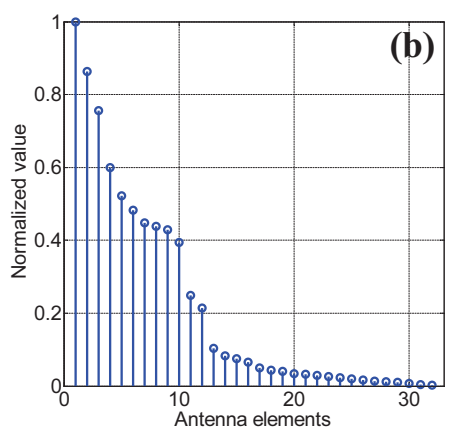

(b) first gated data $\left(D_{n}=20\right)$

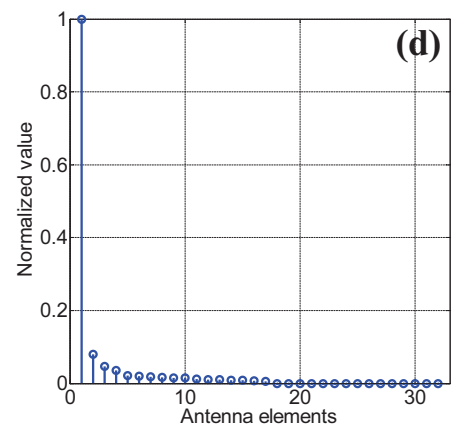

(d) third gated data $\left(D_{n}=31\right)$

Figure 7: Singular value distributions. 


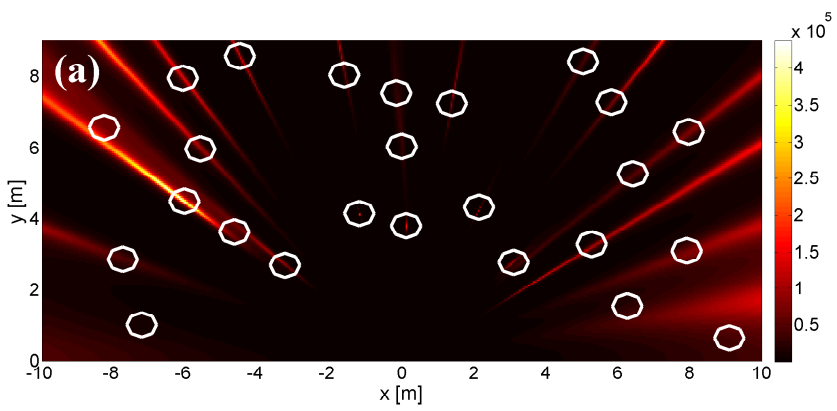

(a) raw data

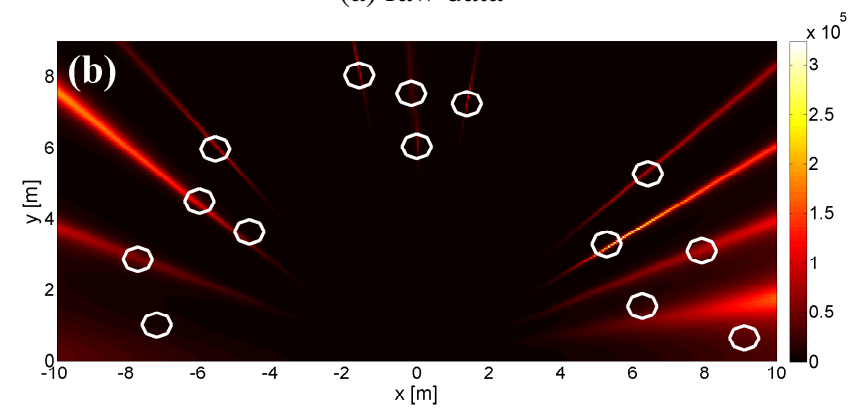

(b) first gated data

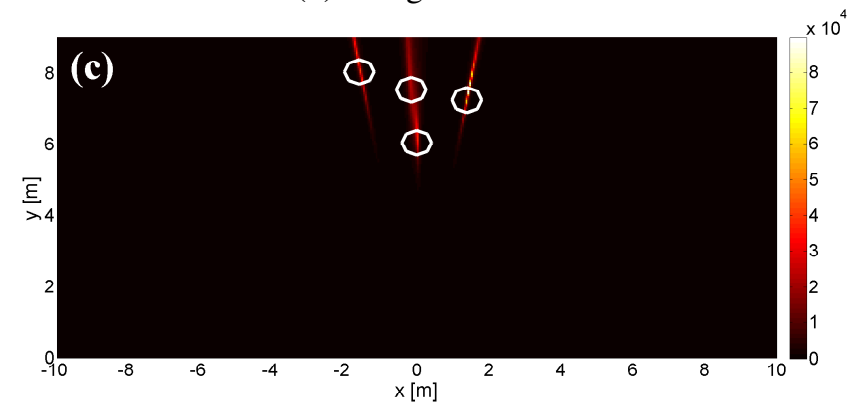

(c) second gated data

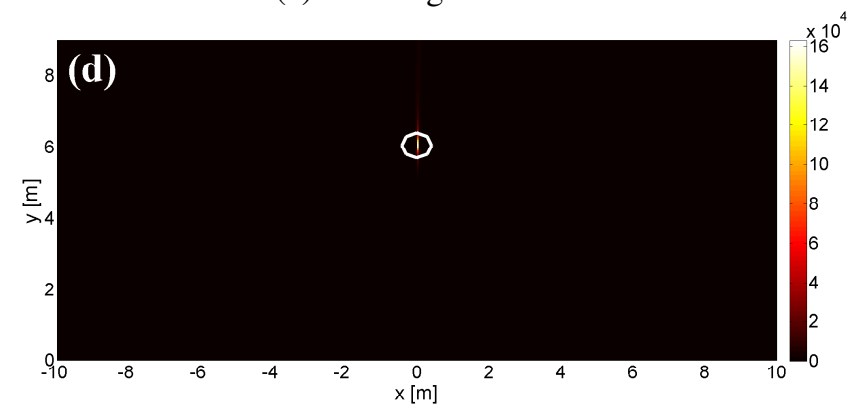

(d) third gated data

Figure 8: Images of the TR-MUSIC pseudo-spectra. 